\title{
Warm water vapor envelope in the supergiants $\alpha$ Ori and $\alpha$ Her and its effects on the apparent size from the near-infrared to the mid-infrared
}

\begin{abstract}
K. Ohnaka
Max-Planck-Institut für Radioastronomie, Auf dem Hügel 69, 53121 Bonn, Germany

Received 12 November 2003 / Accepted 31 March 2004

Abstract. We present a possible interpretation for the increase of the angular diameter of the supergiants $\alpha$ Ori (M 1-2 Ia-Ibe) and $\alpha \operatorname{Her}(\mathrm{M} 5 \mathrm{Ib}-\mathrm{II})$ from the $K$ band to the $11 \mu \mathrm{m}$ region and the high-resolution $11 \mu \mathrm{m}$ spectra without any salient spectral features revealed by Weiner et al. (2003b). The angular diameters as well as the high-resolution spectra of $\alpha$ Ori and $\alpha$ Her obtained in the $11 \mu \mathrm{m}$ region can be reproduced by a warm water vapor envelope, whose presence in $\alpha$ Ori was revealed by Tsuji (2000a) based on the reanalysis of the near-infrared data obtained with the Stratoscope II. While prominent absorption due to $\mathrm{H}_{2} \mathrm{O}$ can be expected from such a dense, warm water vapor envelope, the absorption lines can be filled in by emission from the extended part of the envelope. This effect leads to a significant weakening of the $\mathrm{H}_{2} \mathrm{O}$ lines in the $11 \mu \mathrm{m}$ region, and makes the observed spectra appear to be rather featureless and continuum-like. However, the emission due to $\mathrm{H}_{2} \mathrm{O}$ lines from the extended envelope leads to an increase of the apparent size in this spectral region. The observed angular diameter and the high resolution spectra of $\alpha$ Ori and $\alpha$ Her in the $11 \mu \mathrm{m}$ region can be best interpreted by the water vapor envelope extending to $1.4-1.5 R_{\star}$, with a temperature of $\sim 2000 \mathrm{~K}$ and a column density of $\mathrm{H}_{2} \mathrm{O}$ of the order of $10^{20} \mathrm{~cm}^{-2}$.
\end{abstract}

Key words. infrared: stars - molecular processes - techniques: interferometric - stars: late-type - stars: supergiants stars: individual: $\alpha$ Ori, $\alpha$ Her

\section{Introduction}

Recent mid-infrared interferometric observations have revealed that the diameters of late-type stars such as $\mathrm{M}$ supergiants and Mira variables increase from the $K$ band to the $N$ band. Weiner et al. $(2000,2003 \mathrm{~b}$, hereafter W00 and WHT03b, respectively) observed the supergiants $\alpha$ Ori (M 1-2 Ia-Ibe) and $\alpha$ Her (M 5 Ib-II) at $11 \mu \mathrm{m}$, using the Infrared Spatial Interferometer (ISI) with a narrow spectral bandwidth of $0.17 \mathrm{~cm}^{-1}$. They found that the uniform disk (UD) diameters of these stars are $~ 30 \%$ larger than those measured in the $K$ band, which at least for these stars are considered to approximately represent the continuum radii, as compared to those measured in the optical where scattering due to dust particles is more pronounced (see, e.g., discussion in Dyck et al. 1992). W00 and WHT03b show that the angular diameter of $\alpha$ Ori at $11 \mu \mathrm{m}$ ranges from 53 to 56 mas (43-44 mas in the $K$ band derived by Dyck et al. 1992 and Perrin et al. 2004), while that of $\alpha \mathrm{Her}$ at $11 \mu \mathrm{m}$ is 39 mas (31-32 mas in the $K$ band derived by Benson et al. 1991 and Perrin et al. 2004).

Although dust emission is detected for $\alpha$ Ori, the extended dust shell is unlikely to be responsible for this increase of the diameter for the following reason. The visibility expected for

\footnotetext{
^ e-mail: kohnaka@mpifr-bonn.mpg.de
}

a system consisting of a stellar disk and a very extended dust shell is characterized by a steep drop at low spatial frequencies and a gradual decrease at higher spatial frequencies. The amount of the steep drop at low spatial frequencies corresponds to the flux contribution of the extended dust shell in the field of view, while the stellar disk affects the visibility shape at higher spatial frequencies. The inner radius of the dust shell around M supergiants such as $\alpha$ Ori is derived to be $\sim 50 R_{\star}$ by Danchi et al. (1994). Such a large dust shell is completely resolved with the baselines used by W00 and WHT03b (20-56 m), that is, the dust shell does not affect the shape of the observed visibility function at these baselines. The presence of the dust shell lowers the total visibility at these baselines by an amount equal to the fraction of flux coming from the dust shell in the field of view. W00 and WHT03b take this effect into account in deriving the uniform disk diameters, and show that the observed visibilities can be well fitted by a uniform disk, but with diameters clearly larger than those measured in the $K$ band. For $\alpha$ Her, Danchi et al. (1994) detected a circumstellar dust shell, but more recent spectrophotometric observations by Monnier et al. (1998) show that $\alpha$ Her exhibits very little dust emission. WHT03b suggest that the dust shell around $\alpha$ Her might have evolved since the observations by Danchi et al. (1994). Therefore, the increase of the angular 
diameter observed in $\alpha$ Ori and $\alpha$ Her cannot simply be attributed to the dust shell, while it cannot be completely ruled out that a possible presence of small dust clumps close to the star affects the observed visibility, as Bester et al. (1996) suggest based on their ISI observations of $\alpha$ Ori.

WHT03b also present high-resolution spectra of $\alpha$ Ori and $\alpha$ Her in the same wavelength range as they selected for the ISI observations. These high-resolution spectra were obtained with the TEXES instrument mounted on the Infrared Telescope Facility with a spectral resolution of $\sim 10^{5}$ (Lacy et al. 2002). A glance of these TEXES high-resolution spectra (Fig. 2 of WHT03b) reveals that neither $\alpha$ Ori nor $\alpha$ Her shows any significant spectral features in the bandpasses used for the ISI observations. WHT03b conclude that the interferometric observations with ISI were carried out in the bandpasses which exhibit continuous spectra free from any molecular and/or atomic features. That is, the diameters measured in the $11 \mu \mathrm{m}$ region which appears to be the continuum are significantly larger than the $K$ band diameters, which are also considered to be rather close to the diameter measured in the continuum. Therefore, the interferometric and spectroscopic observations by WHT03b pose a serious problem in interpreting the increase of the angular diameter from the near-infrared to the mid-infrared. As possible explanations for these apparently inconsistent results, WHT03b suggest that the density stratification may be very nonhydrostatic near the photosphere and/or that the angular diameters measured in the near-infrared may be affected by a presence of hot spots, which may make the apparent size of a star smaller than it is.

Tsuji (2000a, hereafter T00a) discovered a warm water vapor layer around two supergiants $\alpha$ Ori and $\mu$ Cep based on the reanalysis of the near-infrared spectra obtained with the Stratoscope II. The presence of water vapor in $\alpha$ Ori was further confirmed by the detection of weak $\mathrm{H}_{2} \mathrm{O}$ absorption in the $6 \mu \mathrm{m}$ spectrum obtained with the Infrared Space Observatory (ISO) (Tsuji 2000b). T00a also studied the pure-rotation lines of $\mathrm{H}_{2} \mathrm{O}$ identified in the high-resolution spectrum of $\alpha$ Ori at $12 \mu \mathrm{m}$ obtained by Jennings \& Sada (1998). Although the observed equivalent widths of the water lines at $12 \mu \mathrm{m}$ are larger than those predicted from the photospheric model of T00a by a factor of $2-3$, the $12 \mu \mathrm{m}$ water lines are expected to be even stronger than observed, if the column density of water molecules derived from the near-infrared spectrum is correct. T00a and Tsuji (2000b) point out the possibility that the absorption may be filled in by emission from the extended part of the water vapor envelope.

If the warm water vapor envelope is present and the emission from it can make the $12 \mu \mathrm{m} \mathrm{H}_{2} \mathrm{O}$ lines appear to be weaker by the filling-in effect, it may also be the case for the $11 \mu \mathrm{m}$ lines presented in WHT03b. That is, the absorption is filled in by the emission resulting from the geometrical extension of the warm molecular envelope, making the spectra almost featureless. While such featureless mid-infrared spectra may not show any hint of the presence of the warm molecular envelope and manifest themselves as the continuum, the extended, warm molecular envelope can cause the apparent diameter in the midinfrared to be larger than the photospheric diameter. Therefore, the warm water vapor envelope may explain the increase of

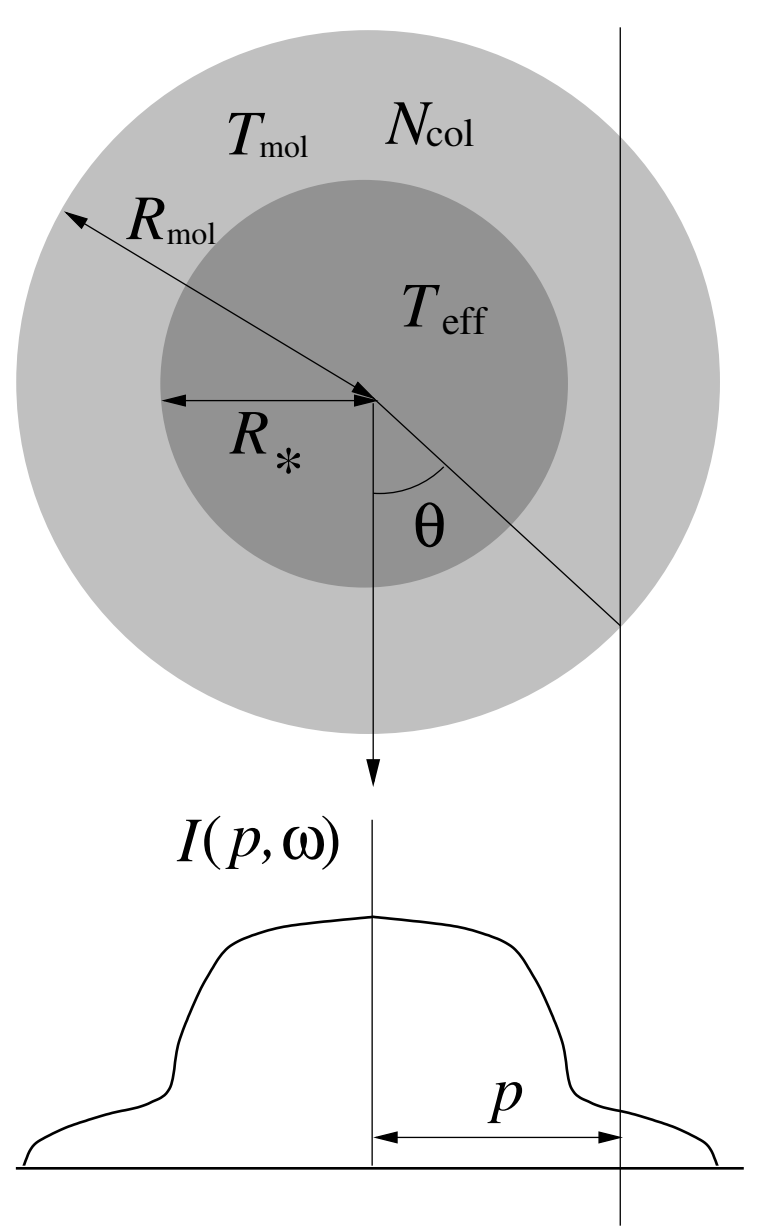

Fig. 1. The model used in the present work. The photosphere is approximated with a blackbody of $T_{\text {eff }}$, with a radius $R_{\star}$. The column density in the radial direction $\left(N_{\mathrm{col}}\right)$ and the temperature of $\mathrm{H}_{2} \mathrm{O}$ gas $\left(T_{\mathrm{mol}}\right)$ as well as the radius of the water vapor envelope $\left(R_{\mathrm{mol}}\right)$ are the input parameters.

angular diameters from the $K$ band to the $N$ band, simultaneously with the spectra observed in the $11 \mu \mathrm{m}$ region.

In the present paper, we examine this possibility for $\alpha$ Ori and $\alpha$ Her. We compare angular diameters as well as spectra predicted from a simple model for the warm water vapor envelope with several observational data: $11 \mu \mathrm{m}$ and $12 \mu \mathrm{m}$ highresolution spectra, the ISO spectrum in the $6 \mu \mathrm{m}$ region, and the angular diameters measured at $11 \mu \mathrm{m}$.

\section{Modeling of the warm molecular envelope}

Our model consists of the central star, which is represented by a blackbody of effective temperature $T_{\text {eff }}$, and a warm molecular envelope extending to $R_{\mathrm{mol}}$, as depicted in Fig. 1 . As the figure shows, the inner radius of the molecular envelope is set to be equal to the radius of the star $R_{\star}$. We adopt an effective temperature of $3600 \mathrm{~K}$ for $\alpha$ Ori (Tsuji et al. 1994) and $3200 \mathrm{~K}$ for $\alpha$ Her (Tsuji 1981). The input parameters are the temperature of water vapor gas $\left(T_{\mathrm{mol}}\right)$, its column density in the radial direction $\left(N_{\mathrm{col}}\right)$, and the geometrical extension of the envelope $\left(R_{\mathrm{mol}}\right.$ in units of $\left.R_{\star}\right)$. The temperature and the density of the water vapor are assumed to be constant in the envelope. 
We first calculate the line opacity due to $\mathrm{H}_{2} \mathrm{O}$ using the HITEMP line list (Rothman 1997), with a Gaussian profile assumed. We adopt a velocity of $5 \mathrm{~km} \mathrm{~s}^{-1}$ for the sum of the thermal velocity and the microturbulent velocity. The energy level populations of $\mathrm{H}_{2} \mathrm{O}$ are calculated in local thermodynamical equilibrium (LTE). The validity of LTE can be examined, using order-of-magnitude estimates of collisional and radiative de-excitation rates, as adopted by Ryde et al. (2002). The collisional de-excitation rate $C_{\mathrm{ul}}$ is given by $C_{\mathrm{ul}} \sim N_{\mathrm{H}} \sigma_{\mathrm{ul}} v_{\mathrm{rel}}$, where $N_{\mathrm{H}}$ is the density of $\mathrm{H}$ atoms, which are assumed to be the primary collision partner, $\sigma_{\mathrm{ul}}$ is the cross section, which we approximate with the geometrical cross section, and $v_{\text {rel }}$ is the relative velocity between the $\mathrm{H}$ atoms and $\mathrm{H}_{2} \mathrm{O}$ molecules. As we will show below, the column densities of $\mathrm{H}_{2} \mathrm{O}$ of the warm water vapor envelope in $\alpha$ Ori and $\alpha$ Her are derived to be $2-7 \times 10^{20} \mathrm{~cm}^{-2}$. The radius of the water vapor envelope is derived to be $1.4-1.5 R_{\star}$, which is translated into $\sim 2 \times 10^{13} \mathrm{~cm}$ with a stellar radius of $650 R_{\odot}$ assumed. The number density of $\mathrm{H}_{2} \mathrm{O}$ is then estimated to be $1.0-4 \times 10^{7} \mathrm{~cm}^{-3}$. The ratio of the number density of $\mathrm{H}$ atoms to that of $\mathrm{H}_{2} \mathrm{O}$ molecules expected in chemical equilibrium is approximately $10^{4}-10^{5}$ for the relevant temperatures and densities. Therefore, the number density of $\mathrm{H}$ atoms is estimated to be $1 \times 10^{11}-4 \times 10^{12} \mathrm{~cm}^{-3}$. With a geometrical cross section $\sigma_{\mathrm{ul}}$ of $10^{-15} \mathrm{~cm}^{2}$ and a relative velocity $v_{\text {rel }}$ of $5 \mathrm{~km} \mathrm{~s}^{-1}$ assumed, these number densities of $\mathrm{H}$ atoms lead to collisional de-excitation rates of $\sim 50-2000 \mathrm{~s}^{-1}$. On the other hand, the rate of spontaneous emission can be estimated from the Einstein coefficients $A_{\mathrm{ul}}$. For the $\mathrm{H}_{2} \mathrm{O}$ molecule, the ranges of $A_{\mathrm{ul}}$ are approximately $\sim 10^{-4}-3 \times 10^{2} \mathrm{~s}^{-1}, \sim 10^{-5}-3 \times 10^{2} \mathrm{~s}^{-1}$, and $\sim 10 \mathrm{~s}^{-1}$ for the 11-12 $\mu \mathrm{m}$ region, $K$ band, and $L^{\prime}$ band, respectively. Therefore, for the wavelength regions that we will discuss below, the assumption of LTE is valid for weak and moderately strong $\mathrm{H}_{2} \mathrm{O}$ lines, while non-LTE effects may not be negligible for strong lines. However, a quantitative assessment of nonLTE effects is beyond the scope of the present paper, and we assume LTE for the $\mathrm{H}_{2} \mathrm{O}$ lines considered here.

Once the line opacity is calculated, the intensity distribution at the wavenumber $\omega$ can be calculated as

$I(p, \omega)=B_{\omega}\left(T_{\mathrm{mol}}\right)\left(1-\mathrm{e}^{-\tau_{\omega}}\right)+B_{\omega}\left(T_{\text {eff }}\right) \mathrm{e}^{-\tau_{\omega}} \operatorname{circ}\left(p / R_{\star}\right)$,

where $p$ is the impact parameter, $\tau_{\omega}$ is the optical depth along the ray at $p$, and $\operatorname{circ}\left(p / R_{\star}\right)$ takes a value of 1 for $p<R_{\star}$ and 0 elsewhere (see Fig. 1). In order to obtain the spectrum, the emergent flux at each wavenumber, $F_{\omega}$, is calculated by

$F_{\omega}=2 \pi \int_{0}^{1} I(p, \omega) \mu \mathrm{d} \mu$

where $\mu$ is defined as $\mu=\cos \theta=\sqrt{1-\left(p / R_{\mathrm{mol}}\right)^{2}}$ with $\theta$ defined in Fig. 1. With an appropriate wavenumber interval, we calculate an intensity profile and a flux at each wavenumber. The corresponding (monochromatic) visibility is calculated from this intensity profile using the Hankel transform (two-dimensional Fourier transform for axisymmetric objects).

\section{Comparison with the observed spectra and angular diameters: $\alpha$ Ori}

Using the above model for the warm $\mathrm{H}_{2} \mathrm{O}$ envelope, we compare the synthetic spectra and the visibilities with the observed data, which include the $11 \mu \mathrm{m}$ spectra presented in WHT03b, the $12 \mu \mathrm{m}$ spectra obtained by Jennings \& Sada (1998), the $6 \mu \mathrm{m}$ spectrum in Tsuji (2000b), and the interferometric observations at $11 \mu \mathrm{m}$ obtained by W00 and WHT03b. We change the input parameters $\left(T_{\mathrm{mol}}, R_{\mathrm{mol}}, N_{\mathrm{col}}\right)$, and search for the combination which can simultaneously reproduce these observational results. The range of the input parameters used in the calculations is as follows: $T_{\mathrm{mol}}(\mathrm{K})=2150,2100,2050,2000$, $1950,1900,1800,1700,1600,1400$, and $1200, N_{\mathrm{col}}\left(\mathrm{cm}^{-2}\right)=$ $1 \times 10^{19}, 1 \times 10^{20}, 2 \times 10^{20}, 5 \times 10^{20}, 1 \times 10^{21}$, and $1 \times 10^{22}$, $R_{\text {mol }}\left(R_{\star}\right)=1.3,1.4,1.45,1.5,1.55,1.6$, and 1.7. It has turned out that the aforementioned observational data for $\alpha$ Ori can be best reproduced with $T_{\mathrm{mol}}=2050 \mathrm{~K}, R_{\mathrm{mol}}=1.45 R_{\star}$, $N_{\text {col }}=2 \times 10^{20} \mathrm{~cm}^{-2}$. The uncertainties of these values are estimated by changing the parameters around the best-fit parameter set by small amounts. We estimate that the uncertainties of the gas temperature, the radius, and the column density of $\mathrm{H}_{2} \mathrm{O}$ are $\pm 100 \mathrm{~K}, \pm 0.1 R_{\star}$, and a factor of $\sim 2$, respectively. The gas temperature and the column density of $\mathrm{H}_{2} \mathrm{O}$ molecules derived in the present work are in rough agreement with those derived by T00a. In the following subsections, we will discuss the comparison for each observational data set.

\section{1. $11 \mu \mathrm{m}$ spectra}

We calculate synthetic spectra in the $11 \mu \mathrm{m}$ region with a wavenumber interval of $0.001 \mathrm{~cm}^{-1}$, and then convolve with a Gaussian profile which represents the effects of the instrument and the macroturbulent velocity. The spectral resolution of the TEXES instrument is $10^{5}$, which translates into an instrumental broadening of $3 \mathrm{~km} \mathrm{~s}^{-1}$. Jennings \& Sada (1998) derived a macroturbulent velocity of $12 \mathrm{~km} \mathrm{~s}^{-1}$ for $\alpha$ Ori. Thus, the synthetic spectra are convolved with a Gaussian with a FWHM of $\sqrt{3^{2}+12^{2}}=12.4 \mathrm{~km} \mathrm{~s}^{-1}$, which corresponds to $0.037 \mathrm{~cm}^{-1}$ at $897 \mathrm{~cm}^{-1}$. In order to take the continuous dust emission from the circumstellar dust shell into account, the convolved and normalized spectrum $F_{\omega}$ is diluted as follows:

$F_{\omega}^{\text {diluted }}=\left(1-f_{\text {dust }}\right) F_{\omega}+f_{\text {dust }}$,

where $F_{\omega}^{\text {diluted }}$ is the final spectrum, and $f_{\text {dust }}$ is the fraction of the flux contribution of the circumstellar dust shell. WHT03b derived $f_{\text {dust }} \sim 0.44$ for $\alpha$ Ori at $11 \mu \mathrm{m}$, and we adopt this value.

Figure 2 shows a comparison between the synthetic $11 \mu \mathrm{m}$ spectra from the best-fit model for $\alpha$ Ori and the observed spectra presented in WHT03b. The dots in the figure represent the observed data, which were read off Fig. 2 in WHT03b, while the solid lines represent the synthetic spectra, which are redshifted by $39 \mathrm{~km} \mathrm{~s}^{-1}$ to match the observations (see WHT03b). Since our model is too simple to deal with the complicated physical processes in the outer atmosphere, we cannot expect very good quantitative agreement for individual spectral features. However, Fig. 2 illustrates that the model calculation can reproduce the absence of conspicuous spectral features as 

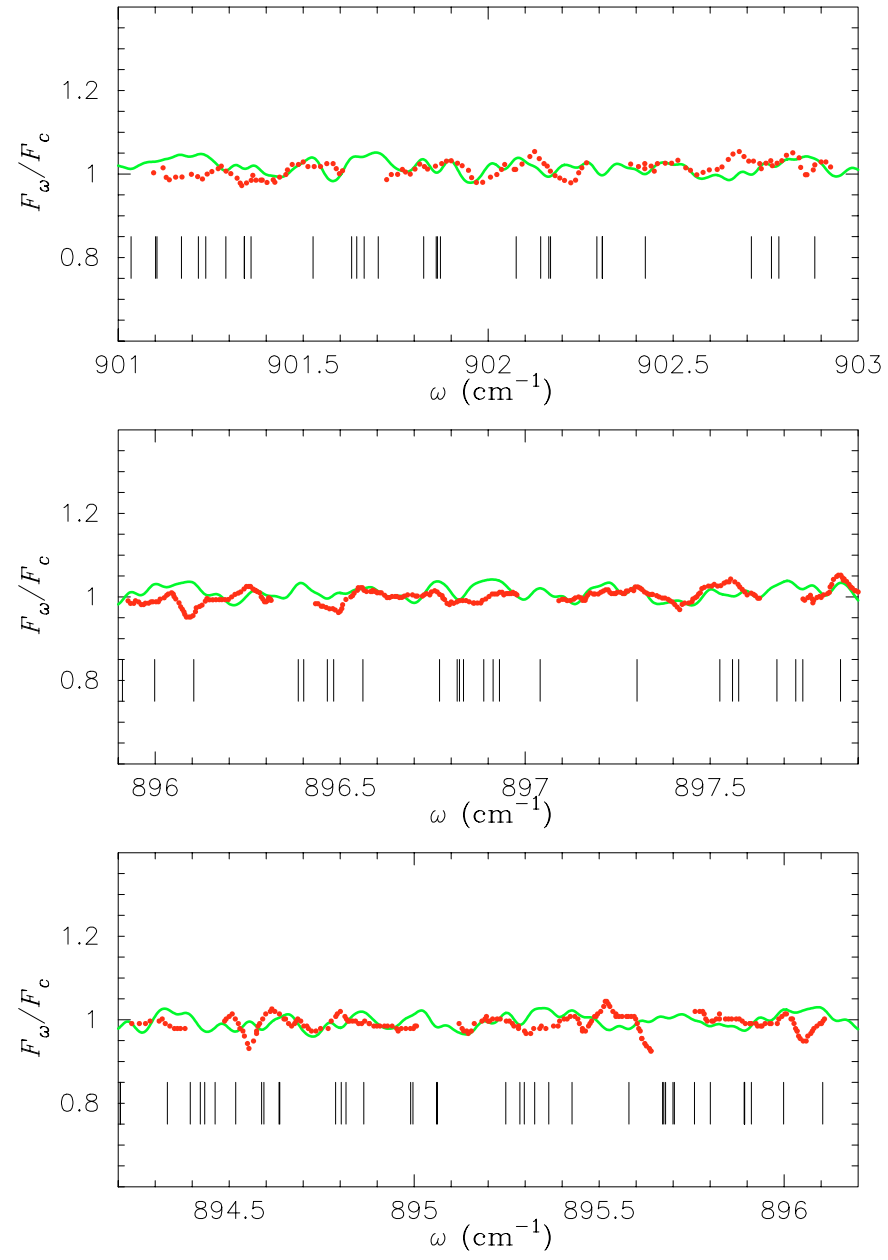

Fig. 2. Spectra in the $11 \mu \mathrm{m}$ region. The dots represent the spectra of $\alpha$ Ori presented in WHT03b, while the solid lines represent the calculated spectra from the best-fit model with $T_{\mathrm{mol}}=2050 \mathrm{~K}$, $R_{\mathrm{mol}}=1.45 R_{\star}$, and $N_{\text {col }}=2 \times 10^{20} \mathrm{~cm}^{-2}$. The synthetic spectra are convolved with a Gaussian with a $F W H M$ of $0.037 \mathrm{~cm}^{-1}$ to account for the effects of the instrument as well as of the macroturbulent velocity, and redshifted by $39 \mathrm{~km} \mathrm{~s}^{-1}$ to match the observations. The positions of the $\mathrm{H}_{2} \mathrm{O}$ lines whose intensity at $2000 \mathrm{~K}$ is stronger than $3 \times 10^{-23} \mathrm{~cm}$ molecule ${ }^{-1}$ are marked with ticks. These line positions are taken from the HITEMP database, and are also redshifted by $39 \mathrm{~km} \mathrm{~s}^{-1}$ with respect to the rest wavenumber.

well as the depths of the small features. Although a number of pure-rotation lines of $\mathrm{H}_{2} \mathrm{O}$ are present as shown with the ticks in the figure, they appear neither as absorption nor as emission. The reason for the absence of salient spectral features is that the absorption lines are filled in by the emission from the outer part of the envelope. In order to illustrate this effect, we show synthetic spectra at the disk center $(\mu=1)$ and near the limb of the warm $\mathrm{H}_{2} \mathrm{O}$ envelope $(\mu=0.3)$ in Fig. 3 (top panel), together with the resulting spectrum (emergent flux) expected for the whole system (middle panel), and the optical depth due to $\mathrm{H}_{2} \mathrm{O}$ in the radial direction (bottom panel). As the bottom panel shows, the optical depth due to $\mathrm{H}_{2} \mathrm{O}$ ranges mostly from $\sim 0.1$ to $\sim 1$ with several peaks reaching $\sim 10$, which means that the warm water vapor envelope is not optically thick. In such a case, the observer see the water vapor
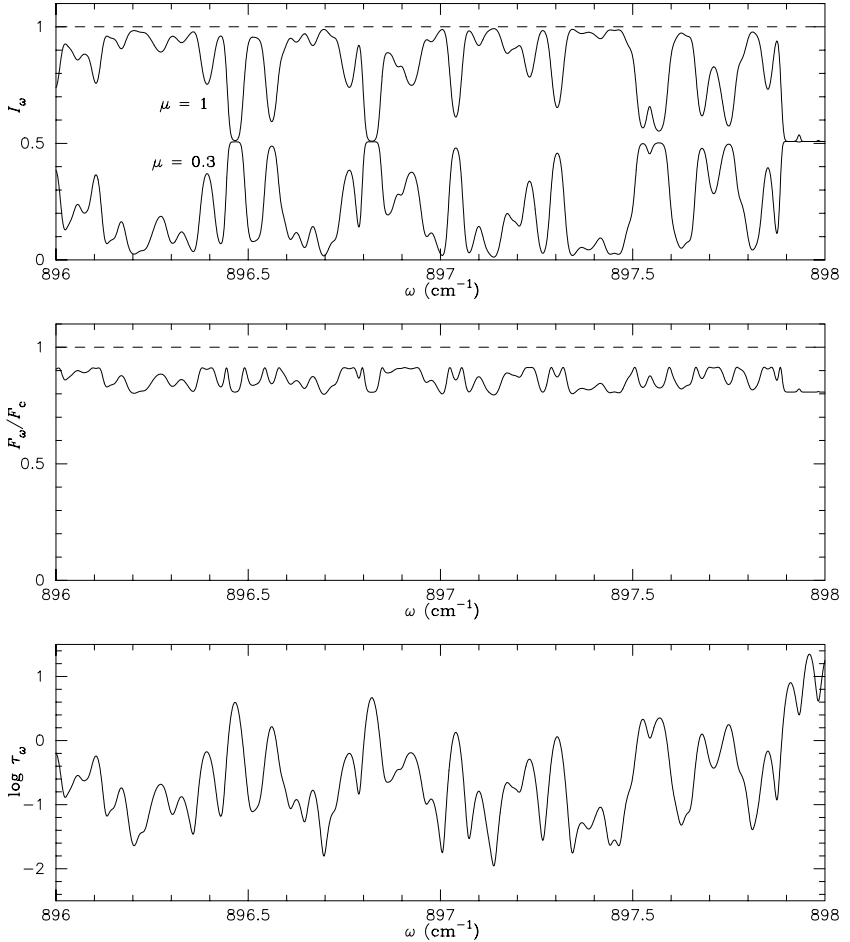

Fig. 3. Top panel: spectra predicted from the best-fit model for $\alpha$ Ori at the stellar disk center $(\mu=1$, upper spectrum) and near the limb of the warm $\mathrm{H}_{2} \mathrm{O}$ envelope $(\mu=0.3$, lower spectrum) in the region around $11.1494 \mu \mathrm{m}$. The continuum level is shown with the dashed line. The flux contribution of dust emission is not included in the spectra shown. Middle panel: the resulting spectrum (emergent flux) expected for the whole object (stellar disk + warm water vapor envelope), calculated from the intensity spectra such as shown in the upper panel, using Eq. (2). Bottom panel: optical depth in the radial direction due to $\mathrm{H}_{2} \mathrm{O}$ lines in the same spectral region. The spectra and the flux shown in the top and middle panels as well as the optical depth shown in the bottom panel are redshifted by $39 \mathrm{~km} \mathrm{~s}^{-1}$ with respect to the rest wavenumber to match the observed spectra shown in Fig. 2, but not convolved with the Gaussian representing the instrumental effect and the macroturbulent velocity.

envelope with the hotter star in the background in a line of sight with $p<R_{\star}$, which leads to appearance of absorption lines. The spectrum at the disk center shown in the top panel illustrates this case, where absorption features are observed at the positions of the $\mathrm{H}_{2} \mathrm{O}$ lines. On the other hand, in a line of sight with $R_{\star} \leq p \leq R_{\mathrm{mol}}$, emission lines are observed, as the spectrum near the limb $(\mu=0.3)$ illustrates. Since the spectrum (i.e. emergent flux) observed for the object (central star + water vapor envelope) is obtained by integrating the intensity over the area that the object projects onto the plane of the sky, the absorption expected from the region with $p<R_{\star}$ and emission expected from the outer region with $R_{\star} \leq p \leq R_{\text {mol }}$ almost cancel out in the resulting spectrum, leading to the nearly featureless spectra as shown in the middle panel.

\section{2. $12 \mu \mathrm{m}$ spectra}

Figure 4 shows the synthetic and observed spectra in the $12 \mu \mathrm{m}$ region. The observed spectra, which were read off Fig. 1 in 

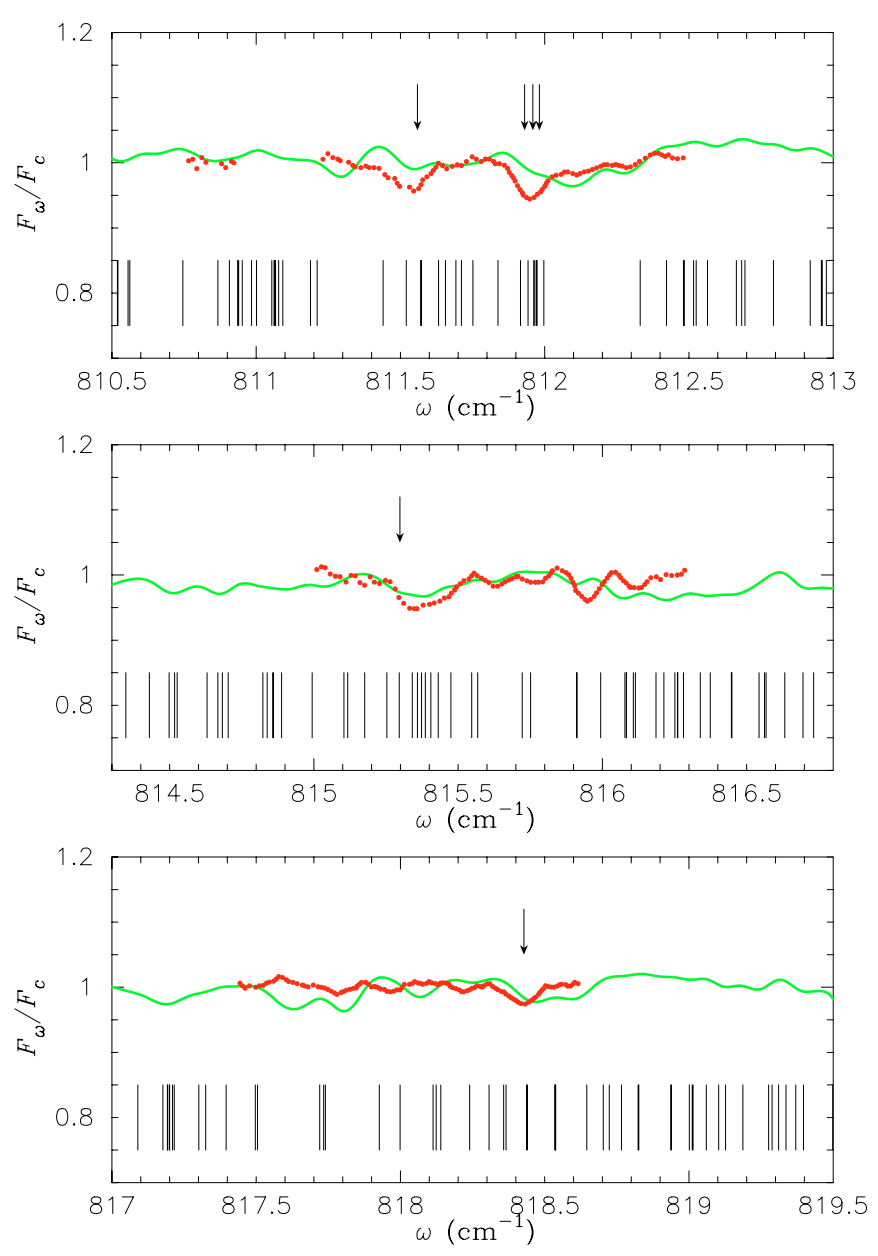

Fig. 4. Spectra in the $12 \mu \mathrm{m}$ region. The dots represent the spectra of $\alpha$ Ori observed by Jennings \& Sada (1998), while the solid lines represent the calculated spectra of the best-fit model. The synthetic spectra are convolved with a Gaussian with a $F W H M$ of $0.1 \mathrm{~cm}^{-1}$ to match the resolution of the spectrometer used by Jennings \& Sada (1998). The ticks represent the positions of $\mathrm{H}_{2} \mathrm{O}$ lines whose intensity at $2000 \mathrm{~K}$ is stronger than $3 \times 10^{-23} \mathrm{~cm}_{\text {molecule }}{ }^{-1}$. The observed and predicted spectra as well as the $\mathrm{H}_{2} \mathrm{O}$ line positions are plotted in the laboratory frame. The $\mathrm{H}_{2} \mathrm{O}$ lines identified by Jennings \& Sada (1998) are shown with the arrows.

Jennings \& Sada (1998), are represented with the dots. The pure-rotation lines of $\mathrm{H}_{2} \mathrm{O}$ identified by Jennings \& Sada (1998) are marked with the arrows in the figure. The synthetic spectra, which are represented with the solid lines, were calculated with a wavenumber interval of $0.001 \mathrm{~cm}^{-1}$, and convolved with a Gaussian with a $F W H M$ of $0.1 \mathrm{~cm}^{-1}$, which corresponds to the resolution of the spectrometer used by Jennings \& Sada (1998). As in the case of the $11 \mu \mathrm{m}$ spectra discussed above, it is necessary to include the dust emission from the circumstellar dust shell in the synthetic spectra. The flux contribution of the dust shell is estimated to be $35 \%$ at $12 \mu \mathrm{m}$ by Jennings $\&$ Sada (1998). Therefore, the calculated spectra are diluted with $f_{\text {dust }}=0.35$. Figure 4 shows that the synthetic spectra can reproduce the absence of strong spectral features, and the observed depths of the weak features are also reproduced to some extent. The filling-in effect due to the outer part of the warm $\mathrm{H}_{2} \mathrm{O}$ envelope weakens the absorption lines significantly,

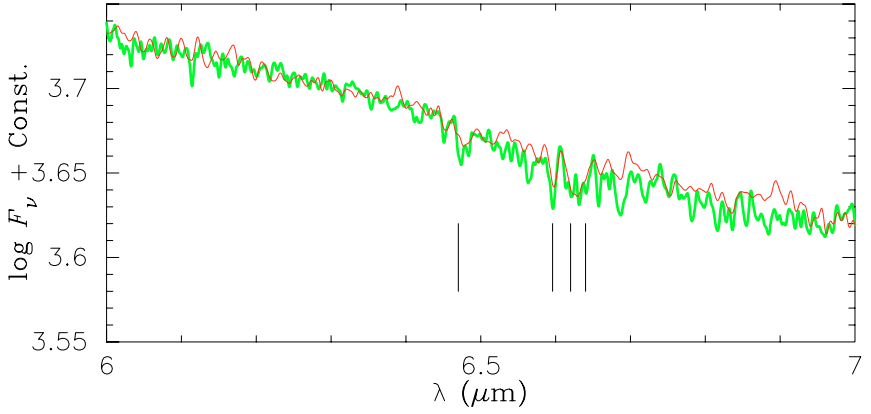

Fig. 5. Spectra in the $6 \mu \mathrm{m}$ region. The thin solid line represents the ISO SWS spectrum of $\alpha$ Ori, while the thick solid line represents the synthetic spectrum predicted from the best-fit model. The synthetic spectrum is convolved with a Gaussian with a $F W H M$ of $1.0 \mathrm{~cm}^{-1}$. The ticks mark the $\mathrm{H}_{2} \mathrm{O}$ absorption features identified by Tsuji (2000b).

as in the case of the $11 \mu \mathrm{m}$ spectra. The absorption features observed at $815.4 \mathrm{~cm}^{-1}$ and $815.95 \mathrm{~cm}^{-1}$ are affected by the blend of $\mathrm{OH}$ lines, which are not included in the present calculation of the synthetic spectra. It should also be noted that T00a shows that the $12 \mu \mathrm{m} \mathrm{H}_{2} \mathrm{O}$ lines may partially originate in the photosphere. If the detailed photospheric structure were incorporated in our model, the $\mathrm{H}_{2} \mathrm{O}$ absorption lines would be stronger than shown in Fig. 4, which might improve the agreement with the observed spectra.

\section{3. $6 \mu \mathrm{m}$ ISO spectrum}

Figure 5 shows a comparison between synthetic spectra and the spectrum obtained with the ISO Short Wavelength Spectrometer (SWS) in the 6-7 $\mu \mathrm{m}$ region where weak absorption due to the $\mathrm{H}_{2} \mathrm{O} v_{2}$ fundamental bands was identified by Tsuji (2000b). The thin solid line represents the spectrum of $\alpha$ Ori (observed on 1997 October 8 UT) retrieved from the ISO data archive, while the synthetic spectrum from the bestfit model, which is convolved with a Gaussian with a FWHM of $1.0 \mathrm{~cm}^{-1}$ to match the resolution of the ISO SWS spectrum ( $R \simeq 1600$ ), is shown with the thick solid line. No dilution due to the circumstellar dust emission is assumed in this wavelength region. Figure 5 demonstrates that the model can fairly reproduce the $\mathrm{H}_{2} \mathrm{O}$ absorption features observed with ISO SWS, which are marked with the ticks in the figure. Therefore, we conclude that our warm $\mathrm{H}_{2} \mathrm{O}$ envelope model is also consistent with the $6 \mu \mathrm{m} \mathrm{H}_{2} \mathrm{O}$ spectrum of $\alpha$ Ori observed with ISO SWS.

\subsection{Angular diameter in the $11 \mu \mathrm{m}$ region}

The comparison between the synthetic spectra and those observed in the $11 \mu \mathrm{m}, 12 \mu \mathrm{m}$, and $6 \mu \mathrm{m}$ regions demonstrates that the warm water vapor envelope extending to $\sim 1.45 R_{\star}$ with a temperature of $2050 \mathrm{~K}$ and an $\mathrm{H}_{2} \mathrm{O}$ column density of $2 \times 10^{20} \mathrm{~cm}^{-2}$ can reproduce the observed spectra. The calculation of the synthetic spectra at $11 \mu \mathrm{m}$ shows that the presence of the warm water vapor envelope can yield a featureless, continuum-like spectrum in the bandpasses used in the ISI observations by WHT03b. If the featureless spectra 
observed at $11 \mu \mathrm{m}$ are a result of the filling-in effect due to the emission from the outer part of the warm water vapor envelope, the angular diameter in this wavelength region can appear larger than the photospheric diameter. This possibility can be examined by computing the visibility from the model intensity profile at each wavelength.

We calculate the intensity profile at a wavenumber interval of $0.001 \mathrm{~cm}^{-1}$, and then from this monochromatic intensity profile, the monochromatic visibility is obtained using the Hankel transform. The monochromatic visibility is convolved with an appropriate response function which represents the spectral resolution of the ISI observations by WHT03b. The spectrally convolved visibility is calculated as follows:

$V_{\text {conv }}(f, \omega)=\int V\left(f, \omega^{\prime}\right) S\left(\omega^{\prime}-\omega\right) \mathrm{d} \omega^{\prime}$,

where $V(f, \omega)$ is the monochromatic visibility at the spatial frequency $f$ and the wavenumber $\omega, V_{\text {conv }}(f, \omega)$ is the spectrally convolved visibility, and $S(\omega)$ is the spectral response function of the bandpass. In the calculation discussed here, $S(\omega)$ is assumed to be a top-hat function with a width of $0.17 \mathrm{~cm}^{-1}$, which is the bandwidth used for the ISI observations. We assume that the $K$-band angular diameter represents the photospheric diameter. Dyck et al. (1992) and Perrin et al. (2004) derived the $K$-band uniform disk diameter of 43-44 mas for $\alpha$ Ori, and we adopt 44 mas as the photospheric diameter. Once the visibility function resulting from the stellar disk and the warm $\mathrm{H}_{2} \mathrm{O}$ envelope (not including the dust shell) is calculated, the uniform disk diameter at each wavenumber can be derived for a given baseline length. We compute the uniform disk diameter for a baseline length of $30 \mathrm{~m}$, which is the mean of the baseline lengths used in the ISI observations by WHT03b.

Figures $6 \mathrm{a}$ and $6 \mathrm{~b}$ show the calculated visibility and the uniform disk diameter of the best-fit model for $\alpha$ Ori in the region around $11.1494 \mu \mathrm{m}$. As mentioned in Sect. 1, the presence of the extended dust shell lowers the visibility by an amount equal to the fraction of the flux contribution of the dust shell. Therefore, the visibility resulting from the stellar disk and the warm $\mathrm{H}_{2} \mathrm{O}$ envelope is lowered by a factor of 0.56 , which accounts for the flux contribution of the stellar disk in this wavelength region. Note, however, that the uniform disk diameter is computed from the visibility excluding the dust shell, because the effect of the presence of the dust shell is already taken into account in the determination of the uniform disk diameters by W00 and WHT03b. Therefore, the uniform disk diameter shown in Fig. 6 can readily be compared with those observationally derived by W00 and WHT03b. As Fig. 6b illustrates, the uniform disk diameter in this spectral region is larger than the photospheric diameter of 44 mas (dotted line in the figure), and the predicted diameter in the ISI bandpasses between 896.7 and $897.0 \mathrm{~cm}^{-1}$ is in agreement with the result obtained by WHT03b.

Figure $6 \mathrm{c}$ shows the intensity profile at the center of the range of the bandpasses used by WHT03b, while Fig. 6d shows the corresponding visibility squared as a function of spatial frequency, together with the observed values presented in Fig. 1 of WHT03b. Figure 6d demonstrates that the predicted visibility squared is somewhat lower that those observed.
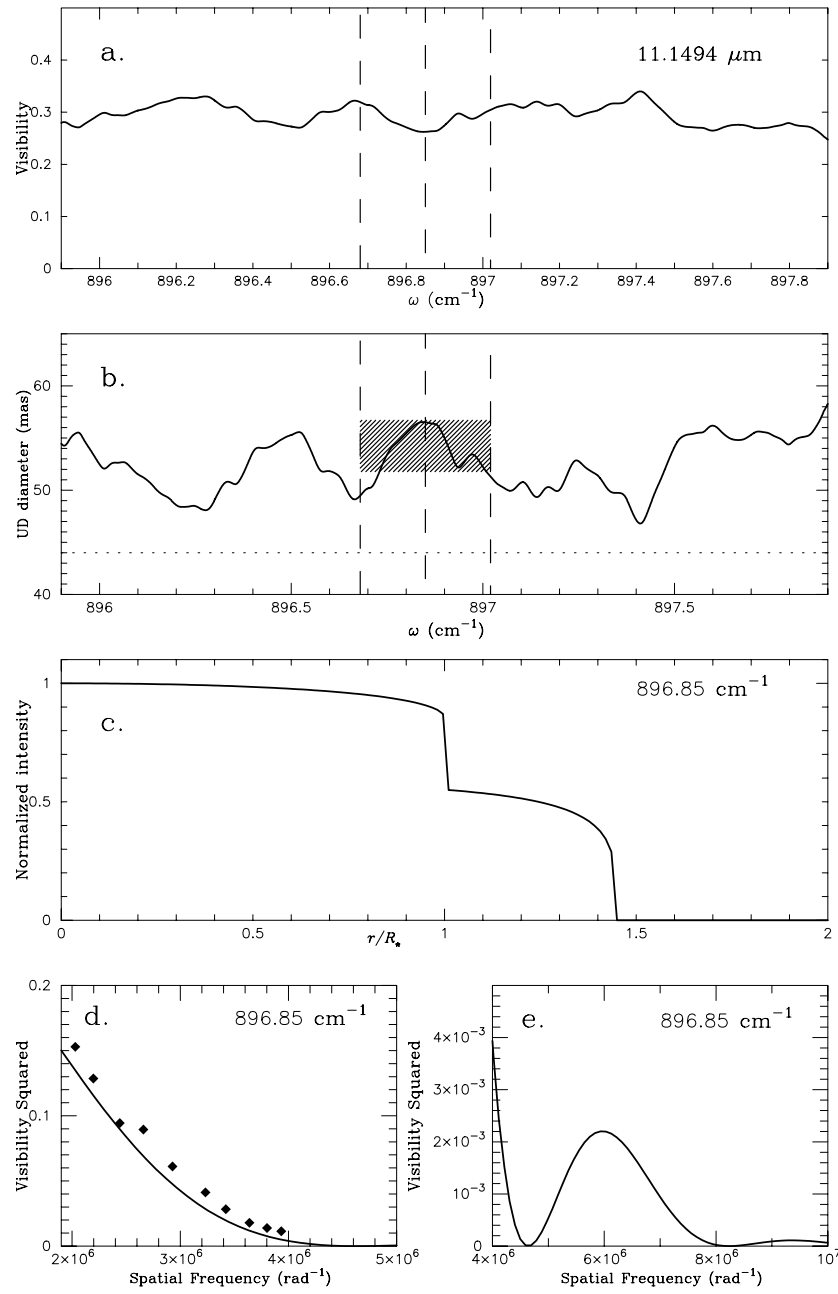

Fig. 6. a) Visibility calculated from the best-fit model for $\alpha$ Ori in the region around $11.1494 \mu \mathrm{m}$. The visibility shown here is calculated for a projected baseline length of $30 \mathrm{~m}$. b) Uniform disk diameter calculated from the spectrally convolved visibility shown in a) for a projected baseline length of $30 \mathrm{~m}$. In a) and b), two bandpasses used by WHT03b are marked with the dashed lines. The bandpasses used for the other observations of $\alpha$ Ori lie between those shown (see WHT03b). The range of the diameters measured in these bandpasses is shown as the hatched region. The dotted line represents the photospheric angular diameter adopted in the calculation. A flux contribution of $44 \%$ from the dust shell is included in the calculations of the model visibility, but not in the computation of the uniform disk diameter. Both plots (a) and b)) are redshifted by $0.117 \mathrm{~cm}^{-1}$ with respect to the rest wavenumber, which corresponds to a radial velocity of $39 \mathrm{~km} \mathrm{~s}^{-1}$, to match the observed spectra shown in Fig. 2. c) The normalized intensity profile at $896.85 \mathrm{~cm}^{-1}$, which is spectrally convolved with the same top-hat function as used in the calculation of the spectrally convolved visibility shown in a). d) The spectrally convolved visibility squared at $896.85 \mathrm{~cm}^{-1}$ (solid line), plotted together with the visibility points observed by WHT03b (filled diamonds). e) The same spectrally convolved visibility squared as in d), but at higher spatial frequencies.

The observed visibility squared can be well fitted with a uniform disk diameter of 52.66 mas (WHT03b), while the predicted uniform disk diameter is 56.6 mas. However, Weiner et al. (2003a) show that the uniform disk diameter measured 
for $\alpha$ Ori fluctuates with an amplitude of $\pm \sim 1.5$ mas, and given this temporal variation of the angular diameter of $\alpha$ Ori on the one hand and the simplicity of our model on the other hand, the agreement between the observed and predicted visibility squared can be regarded as fair. Figure 6e shows the same predicted visibility squared at higher spatial frequencies, which correspond to a projected baseline as long as $\sim 100 \mathrm{~m}$. Observations with such long baselines may be realized by ISI in the near future, and the visibility shape at such high spatial frequencies will be useful for further examining the model for the warm water vapor envelope.

We perform the same calculation for the other two spectral regions observed by WHT03b. Figure 7 shows the predicted uniform disk diameter in the regions around $11.0856 \mu \mathrm{m}$ and $11.1713 \mu \mathrm{m}$. In the $11.0856 \mu \mathrm{m}$ region, the predicted uniform disk diameter ranges from 48 to 53 mas within the bandpass used by WHT03b, which are marked with the dashed lines in the figure. These predicted diameters are systematically lower than the observed value of $54.14 \pm 0.52$ mas. Given the above mentioned temporal variation of the angular diameter of $\alpha$ Ori, however, this slight discrepancy is not regarded as serious disagreement. The predicted diameter in the ISI bandpass in the $11.1713 \mu \mathrm{m}$ region ranges from 51 to 56 mas (lower panel), which is in good agreement with the observed value of $54.20 \pm 0.46$ mas. Thus, our simple model of the warm $\mathrm{H}_{2} \mathrm{O}$ envelope can reasonably explain the observed increase of the angular diameter of $\alpha$ Ori and the spectra in the near-infrared $(6-7 \mu \mathrm{m})$ and in the mid-infrared $(11 \mu \mathrm{m}$ and $12 \mu \mathrm{m})$.

\subsection{Uniform disk diameter in the $K$ and $L^{\prime}$ bands}

While the emission from the warm $\mathrm{H}_{2} \mathrm{O}$ envelope in $\alpha$ Ori can explain the mid- and near-infrared spectra as well as the angular diameter measured with the narrow bandpasses at $11 \mu \mathrm{m}$, a question may arise: if such a dense $\mathrm{H}_{2} \mathrm{O}$ envelope is present, can it affect the angular diameter in the near-infrared? T00a demonstrates that the absorption feature at $1.9 \mu \mathrm{m}$ observed in $\alpha$ Ori can be explained by the warm $\mathrm{H}_{2} \mathrm{O}$ envelope, confirming that the presence of the warm molecular envelope can be detected by detailed stellar spectroscopy in the near-infrared. If the angular diameter measured in the near-infrared were also significantly affected by the warm molecular envelope, our assumption that the $K$-band angular diameter of $\alpha$ Ori represents the photospheric diameter could not be justified, and hence it might not be concluded that the warm $\mathrm{H}_{2} \mathrm{O}$ envelope is responsible for the increase of the apparent diameter from the near-infrared to the mid-infrared. Moreover, the $L^{\prime}$-band angular diameter of $\alpha$ Ori measured by Chagnon et al. (2002) is $42-43$ mas, which is very close to the $K$-band diameter. This suggests that the effect of the warm molecular envelope on the $L^{\prime}$-band diameter should not be prominent.

We examine the effect of the warm $\mathrm{H}_{2} \mathrm{O}$ envelope on the angular diameter measured in the near-infrared by performing the same calculations as in the previous subsection, for the $K$ and $L^{\prime}$ bands. Only $\mathrm{H}_{2} \mathrm{O}$ lines were included in the calculation, and we approximate the $K$ - and $L^{\prime}$-band filter
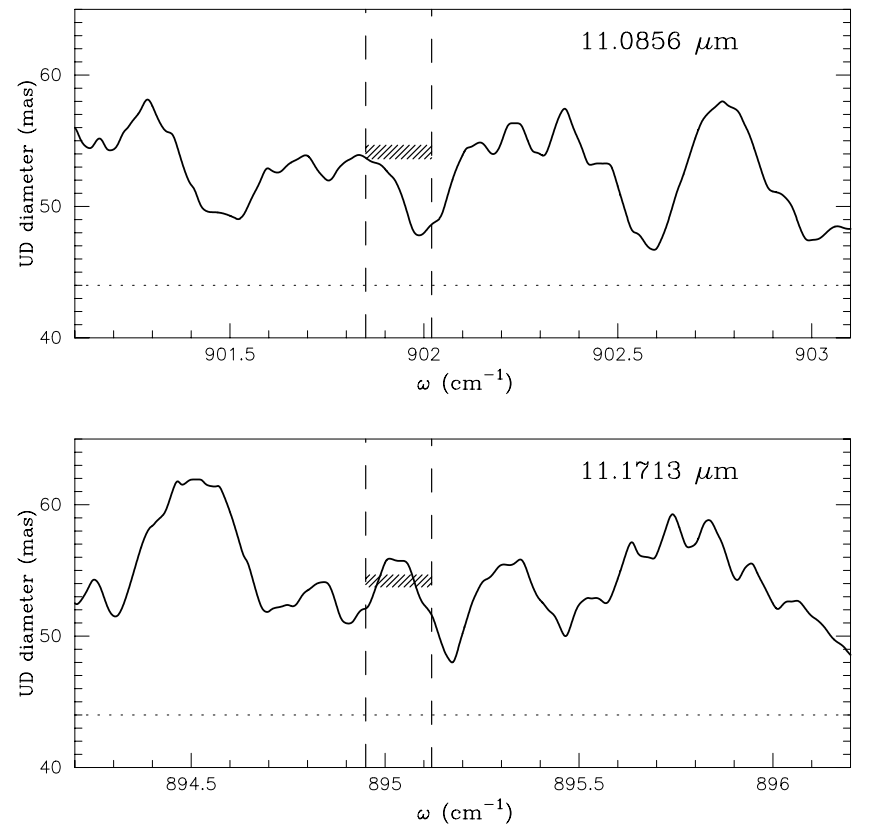

Fig. 7. Uniform disk diameter around $11.0856 \mu \mathrm{m}$ (upper panel) and $11.1713 \mu \mathrm{m}$ (lower panel) calculated from the best-fit model for $\alpha$ Ori. Both plots are redshifted by $0.117 \mathrm{~cm}^{-1}$ in wavenumber, which corresponds to a radial velocity of $39 \mathrm{~km} \mathrm{~s}^{-1}$, to match the observed spectra shown in Fig. 2. The bandpasses used by WHT03b are marked with the dashed lines, and the ranges of the angular diameters measured by WHT03b are represented as the hatched regions. The dotted lines represent the photospheric angular diameter adopted in the calculation.

response functions with top-hat functions centered at $2.15 \mu \mathrm{m}$ with $\Delta \lambda=0.5 \mu \mathrm{m}$ and at $3.8 \mu \mathrm{m}$ with $\Delta \lambda=0.6 \mu \mathrm{m}$, respectively. The uniform disk diameters predicted in the $K$ and $L^{\prime}$ bands have turned out to be 44.5 mas and 45.6 mas, respectively, and these values are very close to the 44 mas which we adopted as the photospheric angular diameter. The predicted angular diameter in the $L^{\prime}$ band is also in agreement with the values derived by Chagnon et al. (2002).

The optical depth due to the $\mathrm{H}_{2} \mathrm{O}$ lines in the $K$ and $L^{\prime}$ bands ranges from $\sim 0.01$ to $\sim 1$ for the gas temperature and the $\mathrm{H}_{2} \mathrm{O}$ column density of the best-fit model $\left(T_{\text {mol }}=\right.$ $2050 \mathrm{~K}$ and $N_{\text {col }}=2 \times 10^{20} \mathrm{~cm}^{-2}$ ), while Fig. 3 shows that the optical depth in the 11.1494 region mostly ranges from $\sim 0.01$ to $\sim 1$. It means that the $\mathrm{H}_{2} \mathrm{O}$ envelope is not yet totally optically thick, and emission from the star can also be observed. In such a case, the effect of emission from the warm $\mathrm{H}_{2} \mathrm{O}$ envelope on the angular diameter is not solely governed by emission from the envelope, but by the intensity ratio between the star and the envelope. With the emission from the star and the warm $\mathrm{H}_{2} \mathrm{O}$ envelope represented with blackbodies of $T_{\text {eff }}=3600 \mathrm{~K}$ and $T_{\text {mol }}=2050 \mathrm{~K}$, respectively, the intensity ratio is expressed as $B_{\lambda}\left(T_{\mathrm{mol}}\right) / B_{\lambda}\left(T_{\text {eff }}\right)$. This intensity ratio is small in the near-infrared, but increases toward longer wavelengths. Therefore, the effect of the warm $\mathrm{H}_{2} \mathrm{O}$ envelope on the angular size is much less prominent in the $K$ and $L^{\prime}$ bands than in the $11 \mu \mathrm{m}$ region. In the $K$-band spectrum of $\alpha$ Ori, the absorption due to $\mathrm{CO}$ and $\mathrm{CN}$ originating in the photosphere is present. However, the effects of these molecular absorption 


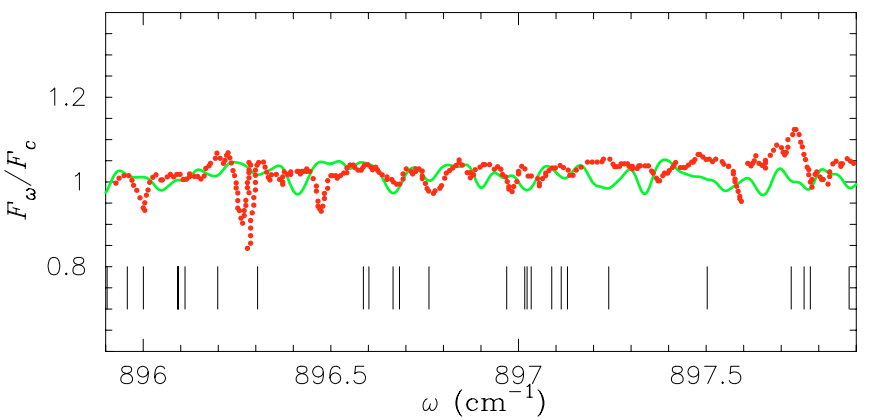

Fig. 8. Spectra in the region around $11.1494 \mu \mathrm{m}$. The dots represent the observed spectrum of $\alpha$ Her, while the solid line represents the synthetic spectrum from the best-fit model. The synthetic spectrum is convolved with a Gaussian with a $F W H M$ of $0.037 \mathrm{~cm}^{-1}$, and blueshifted by $0.084 \mathrm{~cm}^{-1}$ in wavenumber, which corresponds to a radial velocity of $-28 \mathrm{~km} \mathrm{~s}^{-1}$ (see WHT03b), to match the observation. The positions of the $\mathrm{H}_{2} \mathrm{O}$ lines whose intensity at $2000 \mathrm{~K}$ is stronger than $3 \times 10^{-23} \mathrm{~cm}$ molecule ${ }^{-1}$ are marked with ticks. These line positions are taken from the HITEMP database, and are also blueshifted by $28 \mathrm{~km} \mathrm{~s}^{-1}$ with respect to the rest wavenumber.

features on the $K$-band angular diameter are expected to be minor, because the geometrical thickness of the photosphere of $\alpha$ Ori predicted by the classical hydrostatic model is only $9 \%$ of the stellar continuum radius (see T00a), and is much smaller than the radius of the warm $\mathrm{H}_{2} \mathrm{O}$ envelope of $\sim 1.45 R_{\star}$.

\section{Comparison with the observed spectra and angular diameters: $\alpha$ Her}

For $\alpha$ Her, we compare the $11 \mu \mathrm{m}$ spectrum presented in WHT03b and the angular diameter measured in this spectral range. With an effective temperature of $3200 \mathrm{~K}$ adopted for $\alpha$ Her, we search for the combination of the input parameters which can best reproduce the spectra and the angular diameter observed in the $11 \mu \mathrm{m}$ region. The parameters of the best-fit model are found to be $T_{\mathrm{mol}}=2000 \mathrm{~K}, R_{\mathrm{mol}}=1.4 R_{\star}$, and $N_{\text {col }}=7 \times 10^{20} \mathrm{~cm}^{-2}$. We estimate that the uncertainties of the gas temperature, the radius, and the column density of $\mathrm{H}_{2} \mathrm{O}$ are $\pm 100 \mathrm{~K}, \pm 0.1 R_{\star}$, and a factor of $\sim 2$, respectively.

Figure 8 shows a comparison between the observed spectrum (dots) and the calculated one from the best-fit model (solid line). As in the case of $\alpha$ Ori, the synthetic spectrum is convolved with a Gaussian which represents the effects of the instrument as well as of the macroturbulent velocity in the atmosphere of $\alpha$ Her. Tsuji (1988) analyzed the high-resolution spectra of the $\mathrm{CO}$ first overtone bands of $\mathrm{M}$ giants with the use of photospheric models, and detected an absorption excess in the low excitation CO lines, which he concluded originates in the warm molecular envelope. Based on the analysis of this absorption excess, Tsuji (1988) found that the turbulent velocity in the warm molecular envelope of the $\mathrm{M}$ giants in his sample, including $\alpha$ Her, can be as large as $10 \mathrm{~km} \mathrm{~s}^{-1}$. Such a large turbulent velocity is in fact observed in the $12 \mu \mathrm{m}$ spectrum of $\alpha$ Ori, as mentioned in Sect. 3.1. In the present work, we tentatively assume the same macroturbulent velocity in $\alpha$ Her as in $\alpha$ Ori, that is, $12 \mathrm{~km} \mathrm{~s}^{-1}$. Since $\alpha$ Her shows no dust emission feature in the $N$ band (Monnier et al. 1998), no
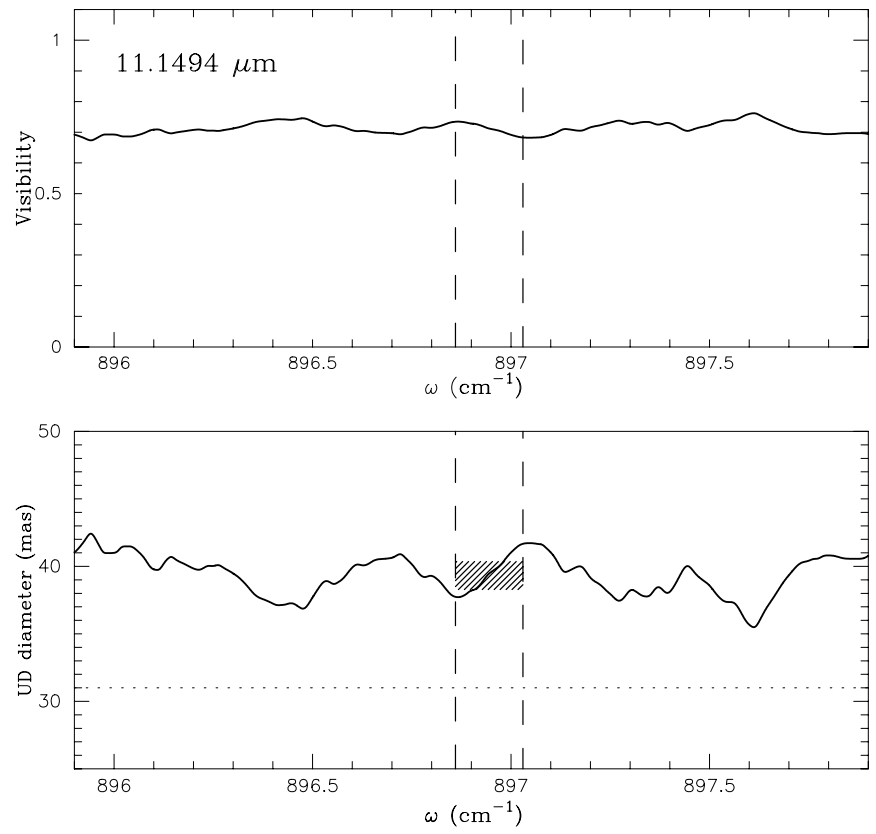

Fig. 9. Visibility and uniform disk diameter calculated from the best-fit model for $\alpha$ Her in the region around $11.1494 \mu \mathrm{m}$. The visibility and the uniform disk diameter shown are calculated for a projected baseline length of $30 \mathrm{~m}$. No flux contribution of the dust shell is assumed in the calculation of the model visibility and uniform disk diameter. The dotted line represents the adopted photospheric angular diameter, while the range of the angular diameter measured by WHT03b is shown as the hatched region. Both plots are blueshifted by $0.084 \mathrm{~cm}^{-1}$ with respect to the rest wavenumber to match the observation.

dilution effect due to dust emission is included in the calculation for $\alpha$ Her. A glance of Fig. 8 reveals that the model can reproduce the observed continuum-like spectrum nearly free from salient features and, to some extent, the depths of the fine spectral features.

We calculate the visibility and the uniform disk diameter in this spectral region. In the calculation of the visibility, we assume that the photospheric angular diameter can be represented by the $K$-band angular diameter. Benson et al. (1991) and Perrin et al. (2004) derived $K$-band angular diameters of 31-32 mas for $\alpha$ Her, and we adopt an angular diameter of 31 mas as the photospheric angular diameter in our calculation. The uniform disk diameter is derived from the calculated visibility for a baseline length of $30 \mathrm{~m}$. Figure 9 shows that the uniform disk diameter is much larger than the photospheric diameter of 31 mas in the spectral region at issue. In the bandpass used in the diameter measurements by WHT03b, which are marked with the dashed lines in the figure, the angular diameter ranges from 38 to 42 mas, which is in agreement with the $39.32 \pm 1.04$ mas measured by WHT03b.

We also examine the effect of the warm $\mathrm{H}_{2} \mathrm{O}$ envelope on the diameter in the $K$ and $L^{\prime}$ bands. The uniform disk diameters in the $K$ and $L^{\prime}$ bands predicted from the best-fit model for $\alpha$ Her are 33.3 mas and 33.9 mas, respectively, and the predicted $L^{\prime}$-band diameter is in agreement with the observed diameter of $31.04 \pm 0.26$ mas derived by Chagnon et al. (2002). This confirms that the $K$-band and $L^{\prime}$-band diameters are not 
significantly affected by the presence of the warm $\mathrm{H}_{2} \mathrm{O}$ envelope, and that the use of the $K$-band diameter as the photospheric diameter is reasonable. It should also be noted that the parameters of the best-fit model for $\alpha$ Her are in rough agreement with those derived by Tsuji (1988) based on the analysis of the absorption excess in the low excitation lines of the $\mathrm{CO}$ first overtone bands.

\section{Discussion}

We have shown that the increase of the angular diameters from the near-infrared to $11 \mu \mathrm{m}$ observed in the supergiants $\alpha$ Ori and $\alpha \mathrm{Her}$ can be explained by the presence of a warm $\mathrm{H}_{2} \mathrm{O}$ envelope, and that dense $\mathrm{H}_{2} \mathrm{O}$ gas with a temperature of $\sim 2000 \mathrm{~K}$ extends to $1.4-1.5 R_{\star}$. These results are in good agreement with those recently obtained by Perrin et al. (2004), who could reproduce the angular diameter of $\alpha$ Ori measured in the $K$ and $L^{\prime}$ bands as well as that at $11.15 \mu \mathrm{m}$, using a spherical gaseous envelope model without a line-by-line calculation of molecular opacities. They derived the temperature and the radius of such a gaseous envelope of $\alpha$ Ori to be $2055 \mathrm{~K}$ and $1.33 R_{\star}$, respectively, with optical depths of $0.06,0.026$, and 2.33 in the $K$-band, $L^{\prime}$ band, and at $11.15 \mu \mathrm{m}$, respectively. Since our model predicts the optical depth as a function of wavenumber, we average the optical depth predicted from our best-fit model for $\alpha$ Ori in the $K$ and $L^{\prime}$ bands. The averaged optical depths of the warm water vapor envelope predicted from our model are 0.05 and 0.026 for the $K$ and $L^{\prime}$ bands, respectively, which is in good agreement with the above values derived by Perrin et al. (2004). For the optical depth in the $11.15 \mu \mathrm{m}$ region, Fig. 3 shows that the optical depth predicted from our best-fit model for $\alpha$ Ori ranges from $\sim 0.01$ up to 10 , which is also in rough agreement with the value derived by Perrin et al. (2004).

From UV observations, $\mathrm{M}$ supergiants are known to have chromospheres as hot as $T_{\mathrm{e}} \sim 8000 \mathrm{~K}$. Gilliland \& Duprees (1996) obtained the first image of $\alpha$ Ori at $2550 \AA$ with the Hubble Space Telescope, and found that the chromospheric extension of $\alpha$ Ori is about 3 times as large as the size measured in the $K$-band. However, the VLA observations of $\alpha$ Ori by Lim et al. (1998) revealed the presence of cooler gas with temperatures of $1300-3400 \mathrm{~K}$ at $2-7 R_{\star}$. Lim et al. (1998) conclude that the hot chromosphere and the cool gas coexist, but with the latter being the more dominant component. Harper et al. (2001) constructed a semiempirical model to explain these VLA observations, and their one-dimensional model has a temperature distribution which first decreases outward from the photosphere, and rises to the maximum of $\sim 3800 \mathrm{~K}$ at $\sim 1.45 R_{\star}$, and then decreases again. While the temperature and the radius of the warm $\mathrm{H}_{2} \mathrm{O}$ envelope we derived for $\alpha$ Ori $(\sim 2050 \mathrm{~K}$ at $\sim 1.45 R_{\star}$ ) seem to be in conflict with this semiempirical model, it should be noted that Harper et al. (2001) suggest that their one-dimensional model might represent the average of an inhomogeneous structure, where the cool gas $(\sim 2000 \mathrm{~K})$ and the hot plasma $(\sim 8000-10000 \mathrm{~K})$ coexist at $\sim 1.45 R_{\star}$. Then it is implied that the warm $\mathrm{H}_{2} \mathrm{O}$ envelope which we modeled in the present work may be part of the cool component detected by the VLA observations and modeled by Harper et al. (2001).
The increase of the angular diameter is detected not only in supergiants but also in Miras and non-Mira M giants. Weiner et al. (2003a) as well as W00 and WHT03b observed the Mira variables $o$ Cet, $\mathrm{R} \mathrm{Leo,} \mathrm{and} \chi$ Cyg with the same observational technique as applied to $\alpha$ Ori and $\alpha$ Her, and found that the angular diameters of these Mira variables are roughly twice as large as those measured in the $K$-band. As in the cases of $\alpha$ Ori and $\alpha$ Her, they used bandpasses which appear not to be contaminated by $\mathrm{H}_{2} \mathrm{O}$ or other spectral lines. However, as we have shown above, the interpretation of the mid-infrared spectra of late-type (super)giants is complicated by flux contribution from the extended outer atmosphere. The increase of diameter is detected not only in the mid-infrared, but also in the $L^{\prime}$ band. Menneson et al. (2002) show that the $L^{\prime}$-band angular diameters of Mira variables as well as semiregular M giants are by a factor of 1.2-2.0 larger than the $K^{\prime}$-band diameters. The increase of the angular diameters from the $K^{\prime}$-band to the $L^{\prime}$-band and the $11 \mu \mathrm{m}$ region observed in the Mira variables and semiregular $\mathrm{M}$ giants may also be explained by the warm molecular envelope, whose presence in these classes of objects is detected by the analyses of infrared molecular spectra (e.g., Tsuji et al. 1997; Yamamura et al. 1999; Cami et al. 2000; Matsuura et al. 2002). This possibility will be further studied in a forthcoming paper (Ohnaka 2004).

Although our ad hoc model for the warm molecular envelope can reproduce the observed spectra as well as the angular diameter increase from the near-infrared to the mid-infrared, it is not a unique solution. The hypothesis of the warm molecular envelope should be further examined by comparing with spectroscopic and interferometric data in other wavelength regions. For example, interferometric observations in many more bandpasses in the $11 \mu \mathrm{m}$ region would provide a more complete picture of the wavelength dependence of the angular diameter, which would be a further constraint for modeling the warm molecular envelope. Interferometry with even higher spectral resolution would also be very useful for testing the hypothesis proposed in the present work. Even if the basic picture of the warm water vapor envelope is correct, our simple model is not sufficient to understand detailed physical properties of the warm molecular envelope such as temperature and density distributions, and therefore, the physical parameters we derived above should be regarded as representative values of the real molecule forming region.

Furthermore, the physical mechanism responsible for the formation of the warm molecular envelope remains to be answered. In the coolest and the most luminous objects such as Mira variables, the levitation of the atmosphere due to stellar pulsation may lead to a density enhancement in the outer atmosphere, and hence creating an environment favorable for molecules to form, as Helling \& Winters (2001) discuss for carbon-rich objects. However, it is not yet clear whether such a mechanism can operate in $\mathrm{M}$ (super)giants with higher effective temperatures and much smaller variability amplitudes. For example, Tsuji et al. (1997), Matsuura et al. (1999), and Tsuji (2001) detected water vapor in the near-infrared spectra of early $\mathrm{M}$ giants and a late $\mathrm{K}$ giant, whose photospheres had been deemed to be too hot for water vapor to form. Ryde et al. (2002) detected $\mathrm{H}_{2} \mathrm{O}$ pure-rotation lines at $11-12 \mu \mathrm{m}$ 
even in the K1.5III giant $\alpha$ Boo. They argue, however, that these $\mathrm{H}_{2} \mathrm{O}$ lines do not originate in the warm molecular envelope, but in the outer layers of the photosphere where temperature may deviate from that predicted by classical, hydrostatic photospheric models, although the mechanism responsible for such a deviation remains ambiguous. In any case, the physical properties of the region between the upper photosphere and the expanding, cold circumstellar envelope have been increasingly probed with various observational techniques. The understanding of physical processes in operation should also be pursued from theoretical point of view to explain the rather common occurrence of the warm molecular envelope, or exactly speaking, the component which cannot be explained in the framework of classical, hydrostatic photospheric models, in a wide range of late-type stars from Mira variables to M (super)giants and $\mathrm{K}$ giants.

\section{Concluding remarks}

Our simple model of the warm $\mathrm{H}_{2} \mathrm{O}$ envelope can simultaneously reproduce the spectra and the angular diameters of $\alpha$ Ori and $\alpha$ Her obtained at $11 \mu \mathrm{m}$. For $\alpha$ Ori, we have also shown that the spectra obtained at $12 \mu \mathrm{m}$ as well as at $6-7 \mu \mathrm{m}$ can be fairly reproduced by this model. The continuum-like spectra of $\alpha$ Ori and $\alpha$ Her observed in the $11 \mu \mathrm{m}$ region can be interpreted as a result of the filling-in due to emission from the outer part of the warm $\mathrm{H}_{2} \mathrm{O}$ envelope. Although the observed featureless $11 \mu \mathrm{m}$ spectra do not show any hint of the presence of the warm $\mathrm{H}_{2} \mathrm{O}$ envelope, it manifests itself as an increase of the angular diameter. Our model can reproduce the increase of the angular diameters observed for $\alpha$ Ori and $\alpha$ Her from the $K$-band to the $11 \mu \mathrm{m}$ region. For $\alpha$ Ori, the gas temperature, the $\mathrm{H}_{2} \mathrm{O}$ column density, and the radius of this $\mathrm{H}_{2} \mathrm{O}$ envelope were derived to be $2050 \mathrm{~K}, 2 \times 10^{20} \mathrm{~cm}^{-2}$, and $1.45 R_{\star}$, respectively. For $\alpha$ Her, we derived a water vapor gas temperature of $2000 \mathrm{~K}$, a column density of $7 \times 10^{20} \mathrm{~cm}^{-2}$, and a radius of $1.4 R_{\star}$.

Acknowledgements. The author would like to thank Prof. T. Tsuji and Dr. T. Driebe as well as the anonymous referee for valuable comments.

\section{References}

Benson, J. A., Dyck, H. M., Mason, W. L., et al. 1991, AJ, 102, 2091

Bester, M., Danchi, W. C., Hale, D., et al. 1996, ApJ, 463, 336

Cami, J., Yamamura, I., de Jong, T., et al. 2000, A\&A, 360, 562

Chagnon, G., Mennesson, B., Perrin, G., et al. 2002, AJ, 124, 2821

Danchi, W. C., Bester, M., Degiacomi, C. G., Greenhill, L. J., \& Townes, C. H. 1994, AJ, 107, 1469

Dyck, H. M., Benson, J. A., Ridgway, S. T., \& Dixon, D. J. 1992, AJ, 104,1982

Gilliland, R., \& Dupree, A. K. 1996, ApJ, 436, L29

Harper, G. M., Brown, A., \& Lim, J. 2001, ApJ, 551, 1073

Helling, Ch., \& Winters, J. M. 2001, A\&A, 366, 229

Jennings, D. E., \& Sada, P. V. 1998, Science, 279, 844

Lacy, J. H., Richter, M. J., Greathouse, T. K., Jaffe, D. T., \& Zhu, Q. 2002, PASP, 114, 153

Lim, J., Carilli, C. L., White, S. M., Beasley, A. J., \& Marson, R. G. 1998, Nature, 392, 575

Matsuura, M., Yamamura, I., Cami, J., Onaka, T., \& Murakami, H. 2002, A\&A, 383, 972

Matsuura, M., Yamamura, I., Murakami, H., Freund, M. M., \& Tanaka, M. 1999, A\&A, 348, 579

Mennesson, B., Perrin, G., Chagnon, G., et al. 2002, ApJ, 579, 446

Monnier, J. D., Geballe, T. R., \& Danchi, W. C. 1998, ApJ, 502, 833

Ohnaka, K. 2004, A\&A, submitted

Perrin, G., Ridgway, S. T., Coudé du Foresto, V., et al. 2004, A\&A, 418,675

Rothman, L. S. 1997, HITEMP CD-ROM (Andover: ONTAR Co.)

Ryde, N., Lambert, D. L., Richter, M. J., \& Lacy, J. H. 2002, ApJ, 580, 447

Tsuji, T. 1981, A\&A, 99, 48

Tsuji, T. 1988, A\&A, 197, 185

Tsuji, T. 2000a, ApJ, 538, 801 (T00a)

Tsuji, T. 2000b, ApJ, 540, L99

Tsuji, T. 2001, A\&A, 376, L1

Tsuji, T., Ohnaka, K., Aoki, W., \& Yamamura, I. 1997, A\&A, 320, L1

Tsuji, T., Ohnaka, K., Hinkle, K. H., \& Ridgway, S. T. 1994, A\&A, 289, 469

Weiner, J., Danchi, W. C., Hale, D. D. S., et al. 2000, ApJ, 544, 1097 (W00)

Weiner, J., Hale, D. D. S., \& Townes, C. H. 2003a, ApJ, 588, 1064

Weiner, J., Hale, D. D. S., \& Townes, C. H. 2003b, ApJ, 589, 976 (WHT03b)

Yamamura, I., de Jong, T., \& Cami, J. 1999, A\&A, 348, L55 